\title{
Facial Overlay Analysis - Combined Repositioning and Computer Alignment
}

\author{
Richard C. ROTH*, Matthew DEPAUW \\ Advanced Imaging and Measurement Laboratory, Analytical Sciences, Amway, Ada MI, USA
}

http://dx.doi.org/10.15221/13.021

\begin{abstract}
Differential comparison of two- and three-dimensional images require aligned subjects in order to discern small structural and contour changes to the facial area. Overlay of subject images at different times and dates is reliant on the ability to precisely align the subject during each clinical visit. The alignment device must be capable of providing sufficient adjustment to accommodate a wide variety of subject body and facial characteristics, and be re-adjusted rapidly and accurately. Changes observed in facial contour are valuable in the analysis of topical cosmetic formulations and in demonstrating efficacy of anti-aging, sagging, and lifting interventions.
\end{abstract}

Stereotactic devices are intended to maintain an orientation of the head for a limited period of time, but not for repeated use. Those employed for surgery are required to prevent movement, but disregard subject imaging. In most imaging alignment devices, restraining alignments encumber the face and neck preventing full image collection by obstruction of view or distortion of facial features. A device to reproducibly position the face and head was previously reported and has been utilized in third party clinical studies.[1] Clinical results have shown positive alignments of subjects, but also identified operational variability associated with subject seating, parameter setting, imaging, training, and subject compliance. The device provided a novel alignment process including, gross subject alignment, fine subject alignment, and direction-of-gaze.

At the baseline clinical visit, a "subject fitting" defines the subject orientation and device parameters utilized during subsequent imaging sessions. Head touch points at the back and top of the head are maintained by the subject and allow undistorted full facial imaging and analysis of the face, jaw, and neck regions. Subject specific device parameters are maintained within 0.1- $0.2 \mathrm{~mm}$. The alignment system includes staff and subject education and training. Subject cooperation and staff oversight are critical in the realignment procedures. Integrated positioning devices reseat the subject body, colocate the head, and maintain the direction-of-gaze of the subject. Overlay comparison results for 3D imaging have indicated excellent correlation of the entire facial region. Combinations of $3 \mathrm{D}$ contour and 2D image overlays were used to evaluate subject profile changes and illustrate locations of facial lifting associated with topical treatments.

Facial overlay routines usually attempt to co-locate the entire facial area for comparison. Full face surface analysis does not separate topically treated and reference regions. Consequently, changes in treated regions are trivialized through averaging the overall facial evaluation. Side-by-side comparison of the inclusive/exclusive region processes demonstrates the need to require separation of treated and non-treated surfaces. Utilizing only reference regions, the treated regions of the face are excluded from the overlay comparison and are considered "experimental surfaces". Only stable untreated facial regions are considered as reference surfaces within the algorithm.

The reference technique maintains the facial reference locations, and allows other non-aligned surfaces to be identified. Movements associated with the neck, upper torso, and shoulders between clinical visits are identified, as well as small facial gestures that might go un-noticed in standard 2D image comparisons. Identification of these movements permits modification of the system and protocol while still capturing full 3D facial contour.

Keywords: 3D, cosmetic, alignment, face

\footnotetext{
* rick.roth@amway.com; 1-616-787-5354; www.amway.com
} 


\section{Introduction}

Comparison of three-dimensional (3D) images illustrates the need for still subjects more easily than with two-dimensional (2D) images. Use of these images to observe facial contour changes, especially small changes, requires precisely aligned subjects. Such changes are observed following efficacious cosmetic product applications; invasive dermatological procedures for anti-aging, sagging, and lifting interventions; and weight loss. Measurements obtained through overlay of 3D images by applying cut planes and topological comparisons are capable of indicating both levels and locations of improvement.

A number of methods have been suggested and are in use for positioning subjects for facial imaging $[2,3]$. Many 2D methods are used to show differences that occur in skin coloration, hyperpigmentation and evenness, lightening, and pore size or number. Some include chin rests, ear bud restraints, and forehead restraints which provide solid reference points for the camera, but do not maintain subject position during realignment $[4,5]$. For these image methods front or side images depicting general facial area and employing color correction are sufficient. The head is placed into an image booth with the chin placed on a chin rest and the forehead placed against a bar (Fig. 1.)(Fig. 2.)(Fig. 3.). In some systems, the chin and forehead rests may rotate for different imaging angles (Fig. 4.).

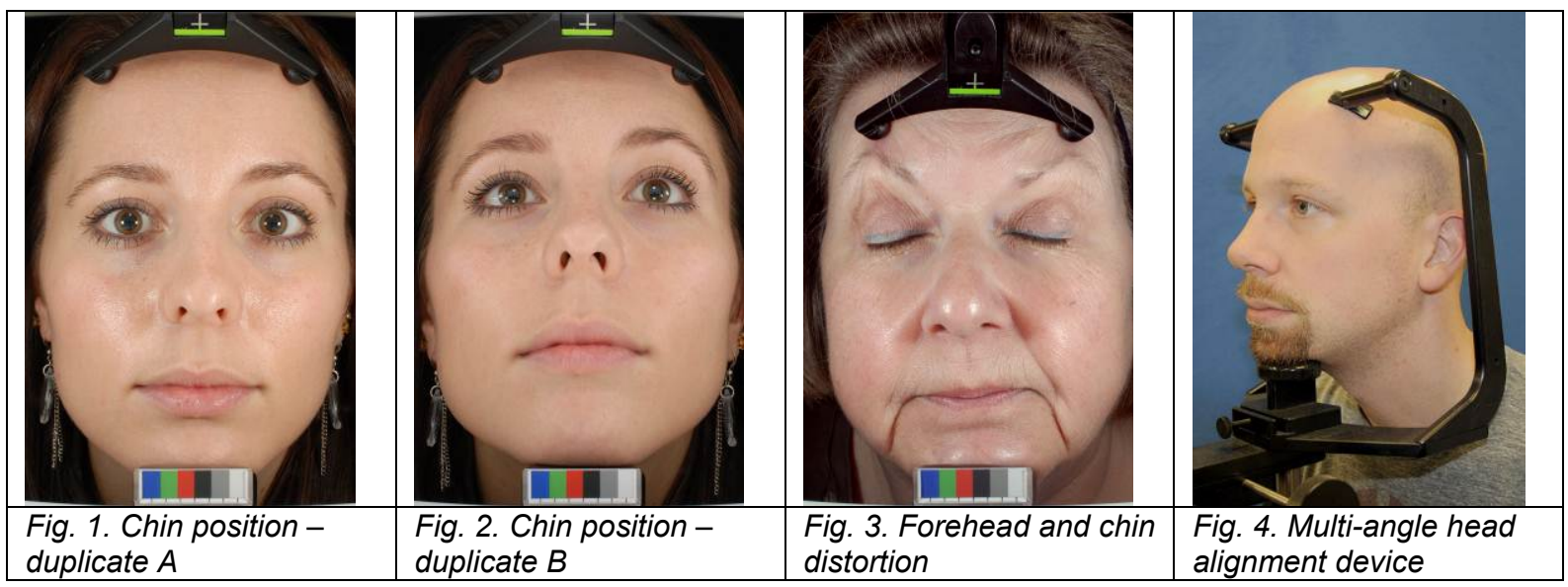

These devices and methods do not take into account repositioning of the subject, restrict or deform the face, and limit view of the head, and neck regions. While the face may be in a similar location, the facial deformations vary per visit and are typically large due to the pressure on the forehead and chin. Many methods rely on rests that obscure portions of the face, do not portray the neck, and the angles of the subject's head/neck are not controlled. "Before and after" images rarely include subject alignment parameter evidence within the image as a verification. Overlay of the subject alignment system and parameters, as well as the subject, within the images provides visual evidence that change in facial contour is due to subject facial change and not from camera or subject orientation differences.

The integrated positioning device: 1) aligned the subject body and head, 2) co-located the head, face and neck region for repeatable analysis, and 3) maintained the subject direction-of-gaze [6]. Device adjustment parameters were recorded and reset for each subject to within 0.1- 0.2 mm. An alignment protocol was previously reported and was used in third party clinical trials involving efficacy of a cosmetic topical application. [1]

\section{Methods and Materials}

\subsection{Alignment principle}

The positioning device, a parallax bar and chair system, provided a novel set of alignment processes including, gross subject alignment, fine subject alignment, direction-of-gaze, and computer adjustment for image overlay. Gross alignment was accomplished through positioning of body and head; fine positioning included centering and leveling; and direction-of-gaze was combined with head alignment integrating the subject to the camera and sensor positions. System parameters were determined during initial "subject fittings" and defined subject-specific locations recorded for subsequent clinical visits. 


\subsection{Subject preparation and education}

At registration, the subjects were provided a fitted soft black v-cut shirt, black headband for pulling hair away from forehead and ears, and a black hair net to assure that stray hair was away from the face. The forehead and ears were visible for imaging and 3D alignment purposes. The use of soft black cloth for the blouse, hair band and hair net assisted in eliminating unnecessary imaging data.

The positioning system permitted unencumbered facial imaging. The alignment processes were designed to minimize subject head tilt, twist, rotation, and depth (from camera) variation. Staff education and subject compliance throughout the processes were critical in obtaining realignment with the imaging system.

Alignment process and education were essential to reduce subject movement (Fig. 5.)(Fig. 6.). Subject understanding of the process and equipment provided greatly reduced time for subsequent subject positioning. Prior to imaging, the subjects viewed a short video presentation on the equipment and alignment process, and were able to review and manipulate a handheld demonstration model of the parallax bar system. The alignment process of seating, body positioning, and head positioning was described to each subject in the imaging room immediately prior to seating. The subject was then realigned three times (as practice) to improve subject understanding of the alignment process prior to image capture.

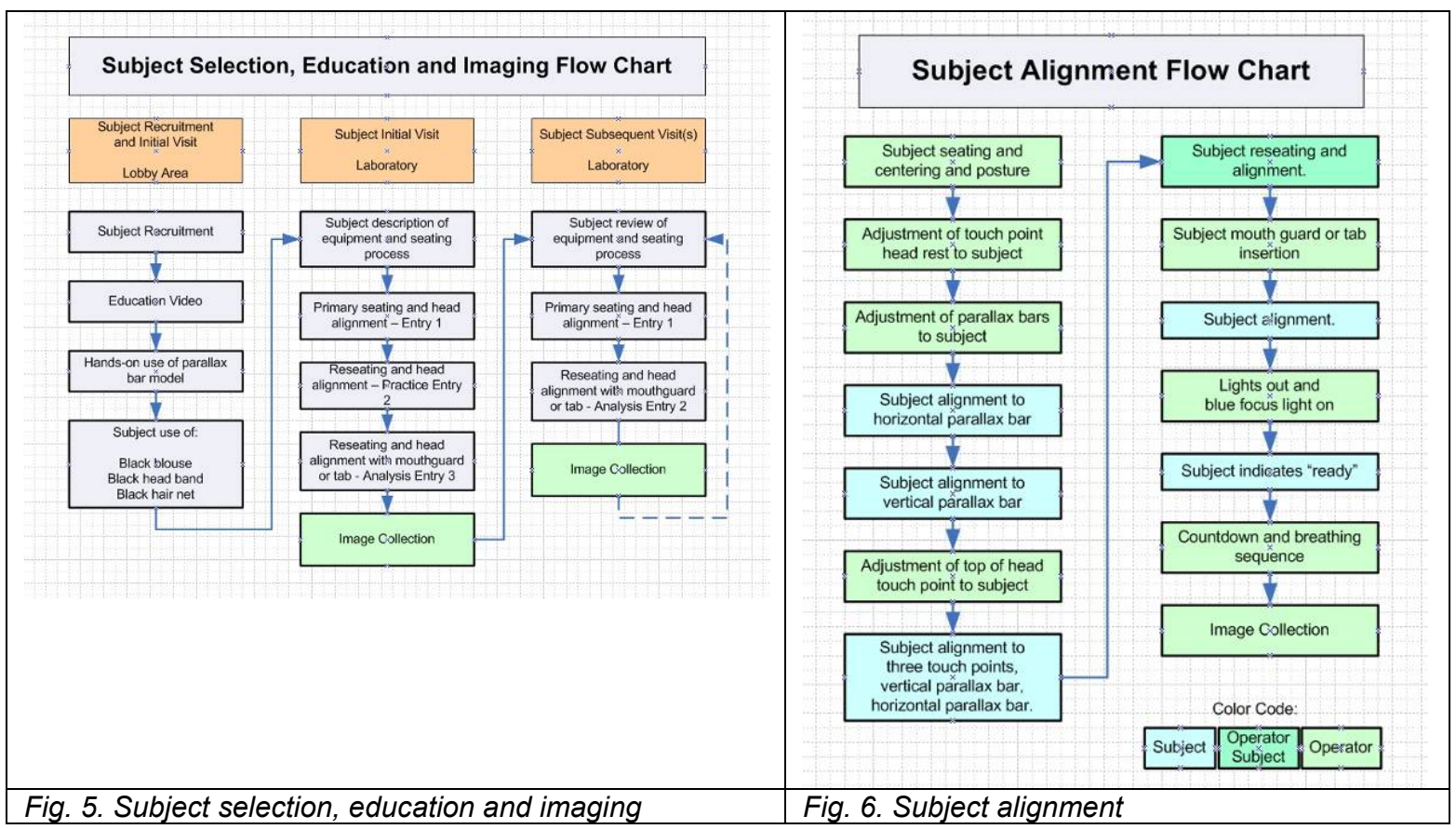

\subsection{Head - body alignment, direction-of-gaze, and parallax bar orientation protocol}

The subjects were aligned following seating in the chair. Feet were placed on a foot-rest integral to the chair. The system parameters were then adjusted to the individual subject including: body position (arm and foot positions); direction-of-gaze (parallax bar height); and head alignment (headrest touch points, top of head touch point, height of head, depth from back support, and neck bar distance.) The 2D and 3D cameras and lighting were integral to the parallax bar to ensure correct alignment.

The entire alignment process including reseating was performed three times for each subject with each visit. The subject was fully removed from the chair for each image within a visit and then reseating and realignment was conducted. As a verification of alignment, and so that a verbal response would not affect position, the subject alignment was confirmed by having the subject raise one index finger when ready. An active frame frontal 2D image overlay, ghosting, was performed to assist the subject in seating alignment at the start of each protocol. 
The operator provided a countdown for subject readiness and the $3 \mathrm{D}$ and $2 \mathrm{D}$ images were immediately obtained. Optional images at adjustable chair rotation angles using détente presets were also obtained. All frontal images were obtained with the eyes open. Eyes were closed for images involving rotation. The subjects were instructed to maintain a neutral expression and keep teeth and jaw in a closed position at all times. The device and alignment process minimized variation in the four axes of co-location: depth, tilt, rotation, and twist. Re-alignment parameters of the device were verified between operators to within 0.1 to $0.2 \mathrm{~mm}$ for all adjustments.

\subsection{Components and construction}

The parallax bar and chair system (Fig. 7.) included a direction-of-gaze parallax bar assembly (Fig. 8.) and chair assembly with headrest (Fig. 9.). The direction-of-gaze parallax bar system consisted of a tripod mount base, a modified enlarger stand mounted on a specialty base interface plate, and an extruded aluminum support structure holding the parallax bars, 2D camera, 3D projectors/sensors, and lighting.

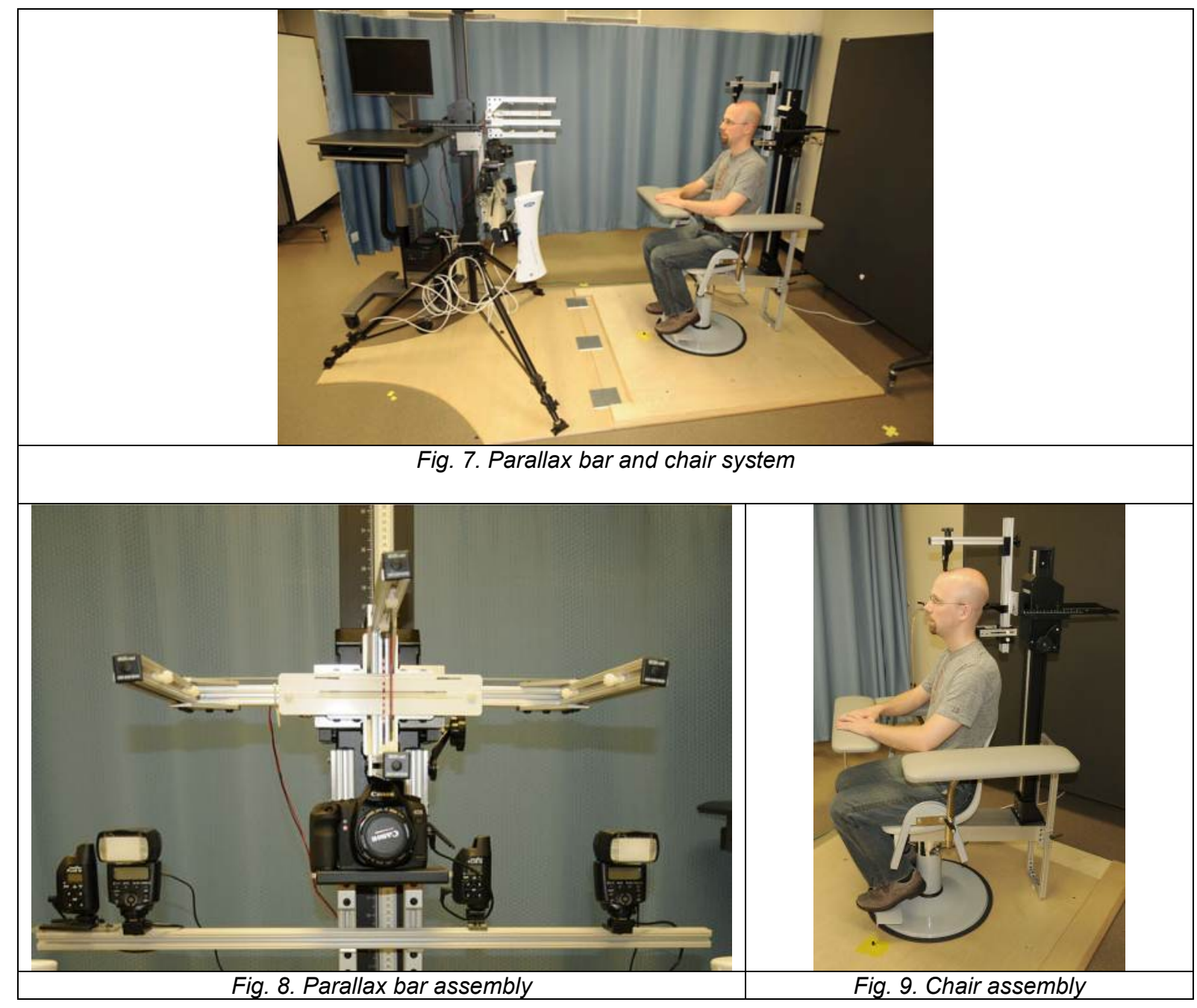

The parallax bar frame and structural supports were constructed of extruded aluminum bars. The parallax bars were constructed of fine polymer wire with a colored polymer coating extended in adjustable tension holders within the extruded aluminum frame. Bar color was selected for contrast and ease of viewing for parallax identification (overlay and centering).

The tripod base supported the height adjustable bracket holding the parallax bars and imaging equipment. The tripod was selected for weight strength and rigid stability. An enlarger copy stand supported the parallax bars and imaging equipment. A second stand, mounted on the chair assembly, supported the headrest alignment brackets. These stands were chosen for range of height adjustability, rigidity, weight capacity and modifiable brackets. 
The 2D/3D - lighting support was constructed from extruded aluminum and routed plate aluminum. The 2D support had a custom camera mount form fit to the camera for ease of removal and realignment. The 3D mount was hinged and contained a location for inserting custom angle solid supports to permit up-angled 3D imaging. The 15-degree angle support was used for this study.

The chair was a custom modified phlebotomy chair centered and mounted to an adjustable floor template. The chair could be rotated to preset angles using an aluminum extension connected to a floor template. The main platform for the template was adjustable to slide forward to accommodate potentially different planes of focus for 3D imaging. The main support stand for the head rest was the enlarger copy stand previously described. The adjustable head, neck and shoulder brackets were custom made from aluminum plate or extruded aluminum.

Two-dimensional digital images were obtained using a Canon 5D Mark II with a Canon $50 \mathrm{~mm}$ f/1.4 lens. Three-dimensional renderings were obtained using a GFM FaceSCAN 3D system with twosensors [3]. Collection and analysis software used was Facexenios 4.3.1 for GFM FaceSCAN 3D. Additional calculations were performed using Microsoft Excel.

The combined parallax bar and chair system was capable of disassembly for shipping and storage in impact resistant shipping cases.

\subsection{Subjects}

Healthy Asian and Caucasian women subjects were recruited by a third party clinical laboratory for a single-center clinical trial over the course of 12 weeks. The product system was defined by use in the facial area (excluding the eye area). The study included regular daily product use during a 12 week application period. Two-dimensional and three-dimensional images were obtained at the baseline, 8 week, and 12 week visits. The study included 72 (36 Asians, 36 Caucasians) recruited subjects meeting the eligibility requirements with a minimum of 60 subjects completing the study. Caucasian women were within age 45-65 and Asian women within age 45-70. Physical parameter requirements included a body mass index (BMI) of $21-28$, with a height requirement in the range $61-77$ inches. Subjects were required to be able to read, speak, write and understand English. Additional eligibility inclusion/exclusion conditions applied. An informed consent form consistent with the requirement in 21 CFR 50.25 was provided to each subject before participation in study procedures.

Subjects that were unable to focus or identify on the parallax bars were excluded from the 3D portion of the study.

\subsection{Analysis}

Typical first use education and "subject fitting" required approximately 10-15 minutes. Subsequent visits required a short review with completion in less than five minutes. The typical subject set/hold time for imaging was less than one minute following subject alignment.

The skin surface was imaged and analyzed using a GFM FaceSCAN 3D with two sensors. The technology is based on the principal of digital stripe projection. Small variations occurring due to noneven skin surfaces are detected by the camera sensor as a deflection and registered. The variations are measured quantitatively and recreated as a 3D rendering from which quantitative measurements may be obtained. Multi-color mapping (topology) provided improved qualitative/quantitative differential viewing of the 3D renderings. The imaging system employed two camera projection/sensors mounted horizontally, which provided overlapping imaging regions of the face and neck. Computer integration of the data sets rendered ear-to-ear 3D images of the face.

\section{Test/Data}

\subsection{Computer overlay of 3D images}

Primary and fine co-location (overlay) was performed through positioning of the subject using the parallax bar and chair system. The 2D and 3D image capture equipment obtain the image files for the analysis. The baseline was designated $t_{0}$ and each subsequent visit as $t_{n}$ with the subscript representing the visit week number. 
Selection of the data file set for 3D analysis was directly dependent on the overlay of the corresponding 2D image set. Closeness of overlay of the 2D image set was accomplished by transparent overlay of each $t_{n}$ onto each $t_{0}$ image. An on/off toggle image alignment algorithm was employed using a three image set from each subject visit resulting in nine comparisons. The closest colocation registration for $a t_{n}$ image (where: $n$ is the visit image set closest in chronological order following baseline) to a $t_{0}$ image defined the specific $t_{0}$ selected image as the baseline reference. This reference $2 \mathrm{D} \mathrm{t}_{0}$ image was used for all subsequent $2 \mathrm{D}$ visit comparisons.

The 3D images directly corresponding in file acquisition time to the selected $2 \mathrm{D}$ image files were defined as the selected $3 \mathrm{D}$ image sets to be used for $3 \mathrm{D}$ facial contour comparison. The $3 \mathrm{D} \mathrm{t}_{0}$ file was defined as the reference file for all subsequent 3D visit comparisons. In this manner, the specific $2 \mathrm{D}$ and $3 \mathrm{D}$ reference files for each subject were determined prior study completion.

Final computer registration of the 3D images was obtained through analysis comparing a baseline image, $t_{0}$, to an image gathered during a subsequent visit, $\left(t_{n}\right)$, designated as either $t_{8}$ or $t_{12}$ representing the eight-week and twelve-week visits, respectively. The image difference $\left(t_{n}-t_{0}\right)$ was the net positional difference in the registered and aligned image set and was reviewed as the colored topographical image.

The 3D rendering registration and comparison process included:

1) Elimination of extraneous background or unrelated components within the capture frame (equipment, reflections, arm skin)(Fig. 10.).

2) Elimination of subject regions outside the facial regions of interest (hairline edge, hanging hair strands, non-contiguous small sections of ear)(Fig. 11.).

3) Coarse registration of the selected comparison images using set point reference selection (bottom center of left and right earlobes, nose tip, upper bridge of nose, nose-philtrum point)(Fig. 12.).

4) Fine registration of the selected comparison images using stable surface selection localized stable surface reference area selection (e.g. lower portion of ears, forehead to zygomatic arch, bridge and end of nose exclusive of nostrils, upper philtrum below nose)(Fig. 13.).

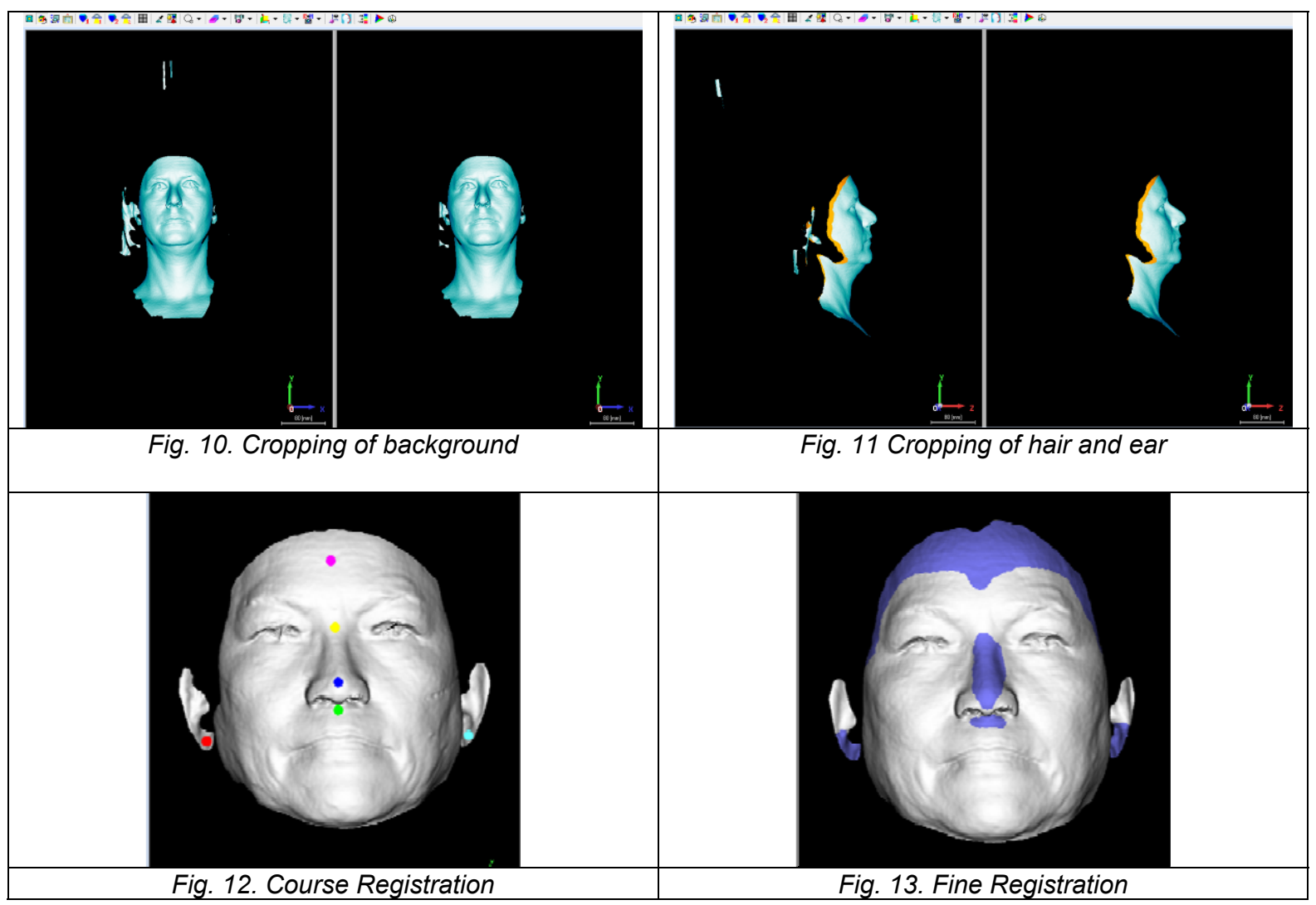


The 3D analysis resulted in whole face and localized topology, and distances between overlays per location. The topology comparison provided information relating to visual goodness-of-fit and subject movement, and distance overlay was specifically obtained from cross sectional analysis. Parallel cross sections were obtained at seven vertical locations (profile, left/right nostril, left/right eye center, left/right outer eye edge)(Fig. 14.); and seven horizontal locations (below eye, nose, nostril, philtrum I, philtrum II, mouth, chin depression, neck at chin)(Fig. 15.); and three diagonal locations (jowl, submental I, submental II)(Fig. 16.). Information obtained from two cross-sections, the horizontal nose and vertical profile positions, is presented here.

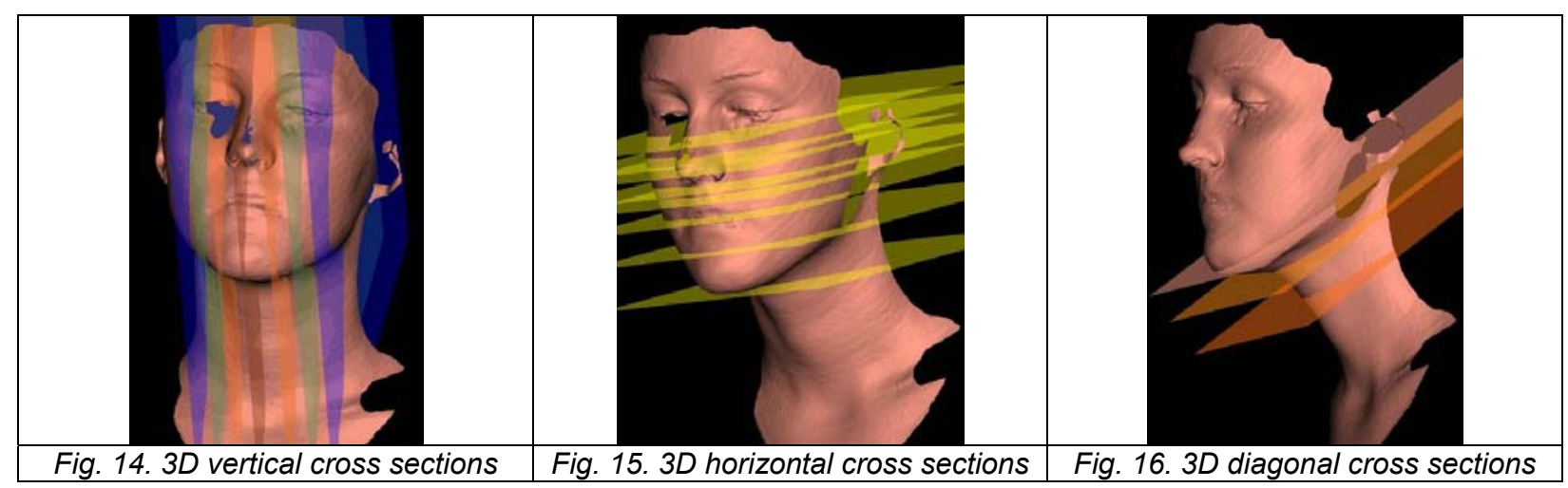

\section{Results}

\subsection{Alignment}

A reference manikin head and neck provided repeatable overlay measurements with a total spatial difference range of $+/-100$ micrometers (above and below average surface). The manikin image overlays were considered best fit results compared to what might be achieved for any human subject (Fig. 17.)(Fig. 18.). Human alignment was not expected to match that of the manikin.

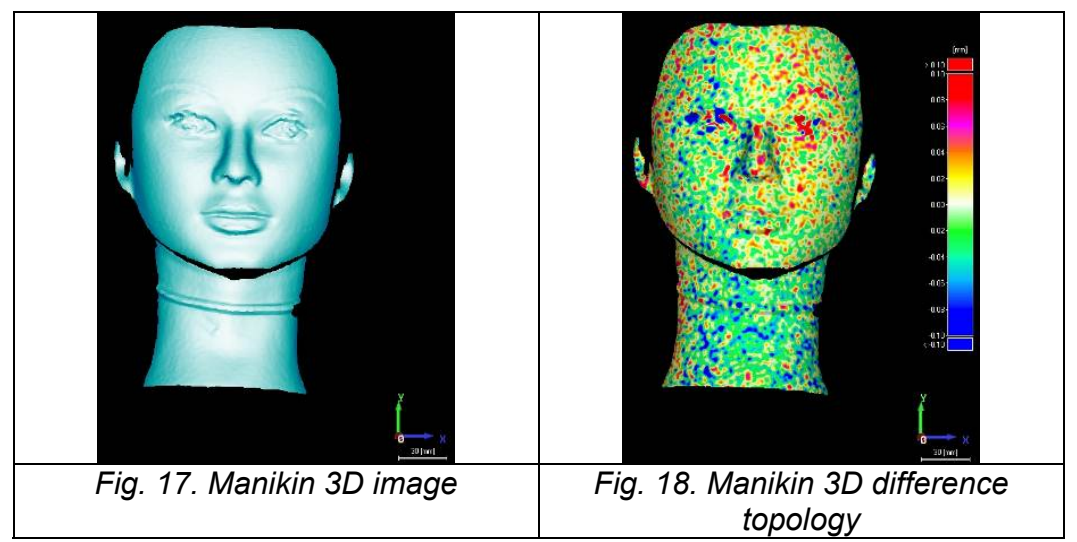

In this study, a cosmetic crème regimen of three products was investigated; a total of 74 subjects were recruited with 67 subjects completing the study. A total of 50 subjects were found to be acceptable for analysis, with 17 subjects deemed unacceptable due to analysis exclusion criteria involving subject movement. Of the 50 subjects, 26 subjects provided positive surface change, 16 subjects negative surface change, with 8 subjects yielding changes less than the minimum change criteria for a single measurement.

Three of the four horizontal nose profile sites (Fig. 19.) showed an average positive surface change $(24,106$, and 123 micrometers) and one site showed an average negative surface change (42 micrometers). The average surface change for all sites was a positive 53 micrometers. The standard deviations of the measurements at the sites were $615,864,616$, and 671 micrometers, respectively. The two regions of stable reference sites on the subject's faces along the vertical profile (main profile) cross section (Fig. 20.) including the entire forehead region, and the full nose bridge, exhibited an average difference of $+/-100$ micrometers (forehead) and $+/-150$ micrometers (nose bridge). These values were obtained at the location of greatest difference within the stable regions. 


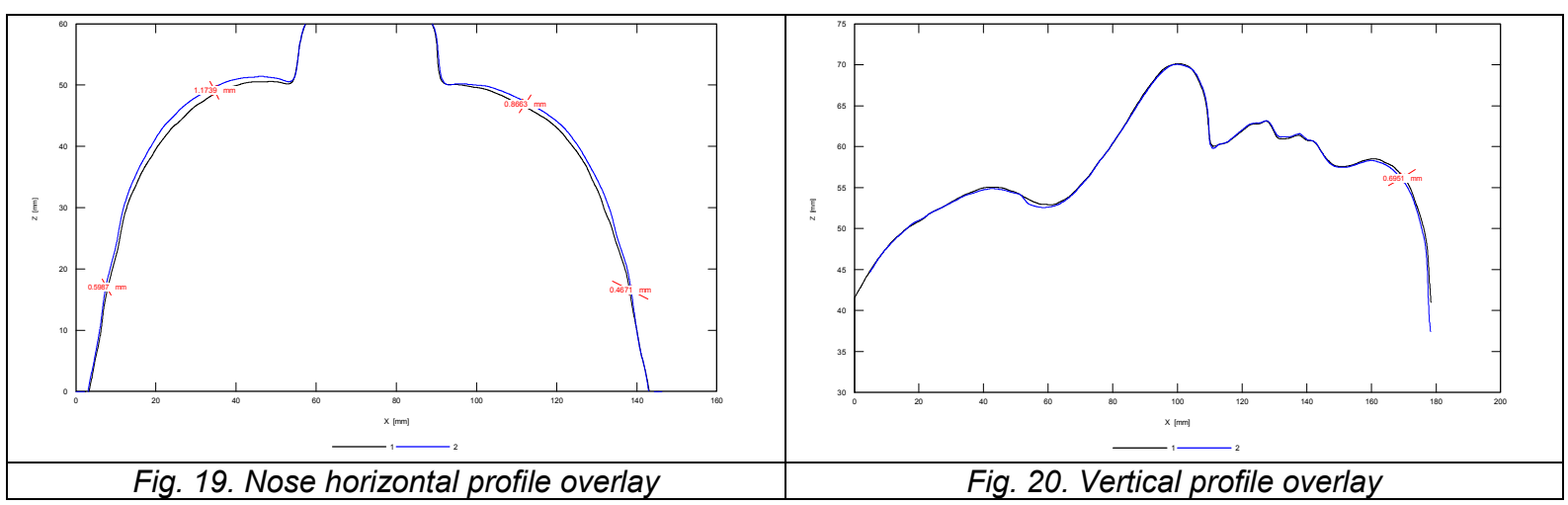

Overlay of the lower component of the same vertical profile from the upper philtrum through the sternal notch was variable. At the sternal notch, the overlay variability was up to $16 \mathrm{~mm}$ for some subjects. This entire region was an immediate indicator of subject compliance for breathing, posture, and maintaining jaw closure during the imaging protocol.

\section{Conclusions and Discussion}

Co-location of the subject was extremely important for image overlay and measurement. The combination of the alignment system, protocol, and computer image algorithm was capable of producing a close overlay of 3D images across a clinical trial timeline of 12 weeks. The overlay was typically less than $1 \mathrm{~mm}$ over the entire face. At stable sites, the overlay was within the range of the reference manikin overlay capability. The overlay difference between aligned human and aligned manikin stable sites was less than had been anticipated and was an indication that the facial overlay process was performing.

These contour changes provide a directional indicator for the horizontal cross section. The statistical variation from baseline to post product use was insufficient to conclude that the $3 \mathrm{D}$ image analysis result directly correlated with clinical grading and consumer/subject product use responses. The vertical profile region across the upper philtrum through the nose and forehead indicated close overlay for the subjects. Post clinical study assessment of the face using stable locations on the nose, forehead and lower ears was considered acceptable for facial image contour comparisons for efficacious cosmetic applications and cosmetic surgery.

Primary subject actions found to influence the alignment were associated with subject noncompliance for incomplete jaw closure, pursing of lips, and smiling. Neck twist and forward position at the head-neck interface and neck-torso interface indicated that subject misalignment in those regions is still significant. Subject pressure against the neck bar modified neck location in the ' $z$ ' (camera) direction that was then exacerbated at the head-neck and neck-torso interfaces. The edge of the eye area provided high image variability from blinking (thickness of eyelid), however, this region is not evaluated during overlay analysis.

Close attention to subject jaw closure is important to maintaining lower facial alignment. Subject expression must be maintained at each clinical visit (Fig. 21.)(Fig. 22.)(Fig. 23.)(Fig. 24.). Changes in facial expression not readily observed during clinical sessions are easily detected during post clinical analysis. Subject and staff education is critical with regard to diligence in adhering to study protocols throughout the image acquisition process. When subjects were actively and frequently reminded to maintain jaw closure, a much greater degree of compliance was observed. 


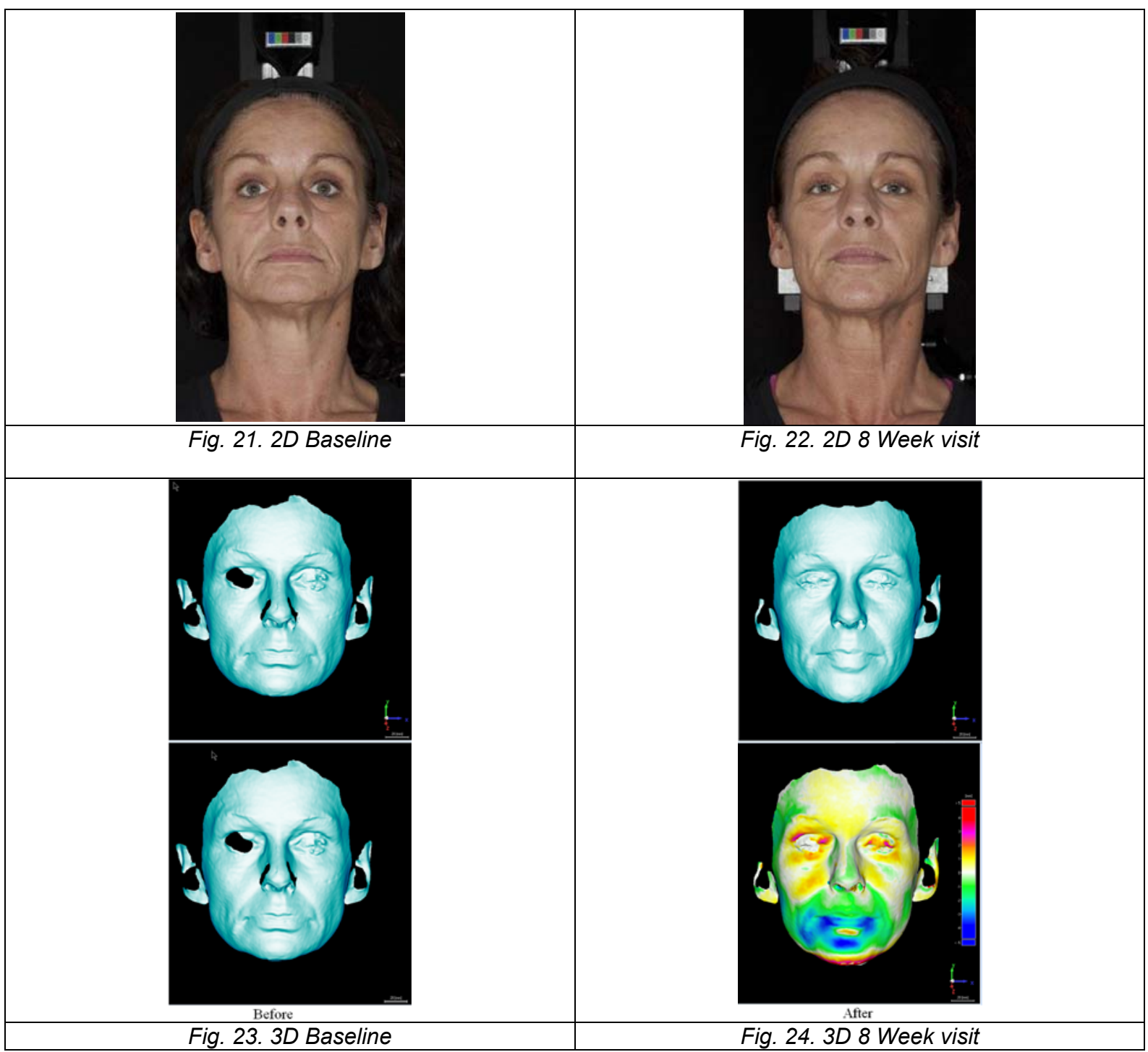

Subject misalignment, particularly in the lower neck area was due to subject seating posture and breathing artifacts. While the head was aligned through the parallax bar system, the neck/torso elements are less strategically aligned. It was clear that greater effort to align the subject, while maintaining the neck region unencumbered, needs to be a priority during future imaging. Based upon the ' $z$ ' axis variations observed in this study, the inclusion of a vertebral and sternal alignment brace system is warranted. The potential to image the neck region separate from the facial region may become necessary in order to attain the required degree of colocation for neck contour evaluation.

Images of the subject that do not include alignment equipment parameters within the frame do not provide an indicator that the subject was equivalently aligned during each image capture. Without this reference it is possible that the surface or contour effect observed may be attributable to other sources such as a change in subject position. It is important that system parameters for head and body alignment are visible in the clinical images for documentation purposes. These settings provide evidence of subject and equipment colocation at baseline and subsequent imaging sessions. For example, a shift was observed in a few images as the baseline and post product visit images were toggled back-and-forth during the overlay process. It could be clearly seen that the parameters had been incorrectly set for realignment even though the parameter settings and training indicated that all set-point values could be reproduced within $\pm 0.1-0.2 \mathrm{~mm}$ and were double checked prior to subject seating. Staff diligence in data recording and the corroboration of settings through the image overlay process ensures that each subject has been positioned and imaged from an identical set of conditions. 
The parallax alignment concept was not clear to all subjects. A number of subjects indicated appropriate alignment with the parallax bars, but their head alignment movements indicated poor understanding of the concept. Several subjects were not able to see the parallax bars clearly. Further inquiry with the subjects found that a laser surgery procedure had altered the focus in the eyes such that one eye focused differently than the other. The language barrier for description of the parallax bar alignment process also proved difficult for several subjects. Those subjects that could not comply with the requirements of alignment protocol were excluded from the study.

In summary, the system design and study results demonstrated a means of obtaining fine overlay of facial areas. The alignment and analysis protocols provided a good method for overlaying $2 \mathrm{D}$ and $3 \mathrm{D}$ images without deformation or encumbrance of the face or neck. The study results indicated the parallax bar and chair alignment system was sufficient for alignment of most subjects, but not all subjects understood the concept. Subject education and compliance with alignment requirements was essential, particularly regarding jaw closure. Overlay of the baseline and post product images provided good alignment of the face and head. Less than adequate alignment of the head-to-neck and neck-to-torso joints was observed, but was still an improvement over other standard alignment methods and did permit image and contour data acquisition. The repeatability of the alignment and analysis protocols indicated close agreement between reference manikins and human subjects such that skin contour changes are capable of being measured through this technique for the face.

\section{References}

1. Roth, R., DePauw, M., and Hepner, A., "Devices and method for precise repositioning of subjects for 3D imaging of head, face, and neck.", Third International Conference and Exhibition on 3D Body Scanning Technologies, Lugano, Switzerland, October 16-17, 2012.

2. Face scanning system "Realscan 3D", 3DDigital Corporation (accessed 2012): www.3ddigitalcorp.com.

3. Face scanning system "FaceSCAN 3D" (accessed 2013): www.gfm3d.com.

4. Whitmer, K., Barford, B., Turner, M., Sullivan, D., and Sommers, M., (2011) "Digital image analysis of facial erythema over time in persons with varied skin pigmentation", Skin Research and Technology, Vol.17, No.3. pp. 348-352.

5. Head positioning system "HeadScan", Orion Concept (accessed 2012): www.orion-concept.com.

6. Roth, R., DePauw, M.,, and Hepner, A., "Devices for Positioning Subjects", U.S. Provisional Patent No. 61/635,592, April 19, 2012. 